\title{
21. INTERSTITIAL WATER STUDIES ON SMALL CORE SAMPLES, DEEP SEA DRILLING PROJECT, LEG $8^{1}$
}

\author{
F. T. Manheim, U.S.G.S., Woods Hole Oceanographic Institute, Woods Hole, Massachusetts \\ and \\ F. L. Sayles, Woods Hole Oceanographic Institute, Woods Hole, Massachusetts
}

\section{INTRODUCTION}

Leg 8 sites are dominated by siliceous-calcareous biogenic oozes having depositional rates of 0.1 to 1.5 $\mathrm{cm} / 1000$ years. Conservative constituents of pore fluids showed, as have cores from other pelagic areas of the Pacific, insignificant or marginally significant changes with depth and location. However, in Sites 70 and 71 , calcium, magnesium and strontium showed major shifts in concentration with depth. These changes appear to be related to recrystallization phenomena in skeletal debris of nannoplankton and to the relative accumulation rate of the sediments.

The chemical anomalies increase relatively smoothly with depth, demonstrating the effectiveness of vertical diffusional communication, and apparent lack of bulk fluid movement, as noted in Leg 7 and other sites.

Analytical methods are similar to those outlined in previous leg reports. We wish to acknowledge the efforts of Charlotte Lawson and Heidi Richards on the laboratory determinations, and the loan of atomic absorption equipment by Derek Spencer of the Woods Hole Oceanographic Institution. Lee S. Waterman joined our group during the course of work on Leg 8, and we thank him for assistance in collation of data for this manuscript. J. D. Milliman, of W.H.O.I., T. C. Moore, Department of Oceanography, Oregon State University, and S. A. Kling of the Cities Service Oil Company contributed useful discussions of the organism-chemical relationships. Thanks are due to S. Honju, of W.H.O.I. for assisting us in searching for unusual chemical composition in some nannoplankton from Leg 8 using a Kevex scanning electron microprobe.

\section{RESULTS}

The chemical analyses of major constituents in the pore fluids are listed in Table 1, which also provide bulk water and $p \mathrm{H}$ data, as supplied from the preliminary shipboard logs. Minor constituents are listed in

\footnotetext{
${ }^{1}$ Contribution No. 2621 of the Woods Hole Oceanographic Institute. Publication approved by the Director, U. S. Geological Survey.
}

Table 2. Replicate samples which were carried through the entire sampling and analytical procedure are shown in parentheses.

The major cations generally demonstrated satisfactory precision. However, occasionally, large discrepancies were apparent in the case of the alkalinities and bicarbonate values calculated from them. We suspect that some absolute loss of alkalinity may have also occurred owing to the nature of the containers (polyethylene pipe) in which the squeezed waters are routinely sealed on board ship. Therefore, the alkalinity determinations are probably correct only for the water samples as received in the laboratory. Significant loss or gain of constituents due to evaporation is unlikely, in view of the consistent and reasonable chloride values obtained. Sodium values are given in two columns in Table 1. Sodium in Column 1 is calculated by summing the anions in terms of milliequivalents per kilogram, subtracting all cations except sodium $(\mathrm{Na})$, and assuming the remainder is due to sodium $(\mathrm{Na})$. That these values are more precise than the directly determined values (Column 2) may be seen by comparing the consistency within holes, both alone and with respect to chloride. Where strontium concentrations are large, as in Holes 71 and 72, strontium is included in the cations for the purpose of calculating sodium $(\mathrm{Na})$. However, the calculated sodium $(\mathrm{Na})$ values are slightly exaggerated owing to the fact that ammonia has not been determined, and it must also contribute a small amount to the total cations.

Significant concentrational relationships and variations with respect to sea (bottom) water include the following:

(1) In Sites 69, 73, 74 and 75 the demonstrated potassium concentrations are irregularly higher than normal, whereas Sites 70 and 71 show marked decreases with depth. At 329 meters in Site 70A, the potassium (K) values have declined to about half sea water values $(0.19 \%)$.

(2) In Sites 69, 73, 74 and 75, calcium and magnesium have relatively minor deviations from their normal sea water concentrations. In Sites 70 and 71, however, strong increases in calcium concentrations 
TABLE 1

Leg 8 Major Constituents of Pore Fluids. Values in $\mathrm{g} / \mathrm{kg}(\% \circ)$, Except as Noted.

\begin{tabular}{|c|c|c|c|c|c|c|c|c|c|c|c|c|c|c|c|c|}
\hline $\begin{array}{c}\text { Sample } \\
\text { Designation }\end{array}$ & $\begin{array}{l}\text { Depth } \\
\text { (m) }\end{array}$ & Age & Description & $\mathrm{Na}^{\mathrm{a}}$ & $\mathrm{Na}^{\mathrm{b}}$ & K & $\mathrm{Ca}$ & $\mathrm{Mg}$ & $\begin{array}{c}\text { Total } \\
\text { Cations } \\
\text { (meq/kg) }\end{array}$ & $\mathrm{Cl}$ & $\mathrm{SO}_{4}$ & $\underset{(\mathrm{meq} / \mathrm{kg})}{\text { Alk. }}$ & $\mathrm{HCO}_{3}{ }^{\mathrm{c}}$ & $\begin{array}{c}\text { Total } \\
\text { Anions } \\
(\mathrm{meq} / \mathrm{kg})\end{array}$ & Sum $^{d}$ & $\mathrm{H}_{2} \mathrm{O}(\%) p \mathrm{H}^{\mathrm{e}}$ \\
\hline
\end{tabular}

Site $69\left(6^{\circ} 00.0^{\prime} \mathrm{N}, 152^{\circ} 51.9^{\prime} \mathrm{W}\right.$, water depth $4978 \mathrm{~m}$, S. of Hawaii)

Hole 69

$\begin{array}{ccllllllllllllllll}3-3 & 27 & \begin{array}{l}\text { Middle } \\ \text { Miocene }\end{array} & \begin{array}{l}\text { Dark yellowish brown } \\ \text { radiolarian ooze w/beds } \\ \text { of nannofossil-radiolarian } \\ \text { ooze. }\end{array} & 10.9 & 10.7 & 0.45 & 0.41 & 1.26 & 601 & 19.64 & 2.67 & 2.7 & 0.16 & 612 & 35.5 & 77 & 7.1 \\ 5-3 & 120 & \begin{array}{l}\text { Lower } \\ \text { Oligocene }\end{array} & \begin{array}{l}\text { Pale yellow brown and } \\ \text { pale brown nannofossil- } \\ \text { radiolarian oozes. }\end{array} & 10.8 & 10.7 & 0.48 & 0.40 & 1.32 & 606 & 19.45 & 2.67 & 3.5 & 0.21 & 608 & 35.3 & 65 & 7.7\end{array}$

Hole 69A

\begin{tabular}{|c|c|c|c|c|c|c|c|c|c|c|c|c|c|c|c|c|c|}
\hline $3-2$ & 81 & $\begin{array}{l}\text { Middle } \\
\text { Oligocene }\end{array}$ & $\begin{array}{l}\text { White to yellow brown } \\
\text { nannofossil-radiolarian } \\
\text { ooze. }\end{array}$ & 11.0 & $\begin{array}{l}10.7 \\
(10.8)\end{array}$ & $\begin{array}{c}0.46 \\
(0.46)\end{array}$ & $\begin{array}{c}0.40 \\
(0.40)\end{array}$ & $\begin{array}{l}1.25 \\
(1.25)\end{array}$ & 602 & 19.47 & 2.94 & $\begin{array}{c}3.1 \\
(3.4)\end{array}$ & $\begin{array}{c}0.19 \\
(0.21)\end{array}$ & 614 & 35.7 & 46 & 7.7 \\
\hline $8-6$ & 143 & $\begin{array}{l}\text { Lower } \\
\text { Oligocene }\end{array}$ & $\begin{array}{l}\text { White w/beds of pale } \\
\text { brown nannofossil- } \\
\text { radiolarian ooze. }\end{array}$ & 10.8 & $\begin{array}{c}10.7 \\
(11.0)\end{array}$ & $\begin{array}{c}0.43 \\
(0.46)\end{array}$ & $\begin{array}{c}0.41 \\
(0.40)\end{array}$ & $\begin{array}{c}1.27 \\
(1.25)\end{array}$ & 607 & 19.44 & 2.63 & $\begin{array}{c}1.6 \\
(2.4)\end{array}$ & $\begin{array}{c}0.07 \\
(0.15)\end{array}$ & 605 & 35.0 & 48 & 7.7 \\
\hline $11-2$ & 216 & $\begin{array}{l}\text { Middle } \\
\text { Eocene }\end{array}$ & $\begin{array}{l}\text { Dark brown radiolarian } \\
\text { ooze. }\end{array}$ & 11.0 & $\begin{array}{c}10.6 \\
(10.8)\end{array}$ & $\begin{array}{c}0.41 \\
(0.41)\end{array}$ & $\begin{array}{c}0.39 \\
(0.41)\end{array}$ & $\begin{array}{l}1.25 \\
(1.25)\end{array}$ & 599 & 19.54 & 2.72 & $\begin{array}{c}2.9 \\
(2.8)\end{array}$ & $\begin{array}{l}0.18 \\
(0.17)\end{array}$ & 611 & 35.5 & 62 & 7.6 \\
\hline
\end{tabular}

Site $70\left(6^{\circ} 20.1^{\prime} \mathrm{N}, 140^{\circ} 21.7^{\prime} \mathrm{W}\right.$, water depth $5059 \mathrm{~m}$, continuation of north to south profile started on Leg 5)

Hole 70

\begin{tabular}{|c|c|c|c|c|c|c|c|c|c|c|c|c|c|c|c|c|c|}
\hline $3-1$ & 17 & $\begin{array}{l}\text { Middle } \\
\text { Miocene }\end{array}$ & $\begin{array}{l}\text { Dark grayish brown radio- } \\
\text { larian ooze. }\end{array}$ & 10.9 & $\begin{array}{c}10.8 \\
(10.7)\end{array}$ & $\begin{array}{c}0.44 \\
(0.44)\end{array}$ & $\begin{array}{c}0.46 \\
(0.45)\end{array}$ & $\begin{array}{c}1.23 \\
(1.21)\end{array}$ & 602 & 19.52 & 2.57 & $\begin{array}{c}3.6 \\
(3.5)\end{array}$ & $\begin{array}{c}0.22 \\
(0.21)\end{array}$ & 608 & 35.3 & 32 & 7.5 \\
\hline $7-2$ & 55 & $\begin{array}{l}\text { Lower } \\
\text { Miocene }\end{array}$ & $\begin{array}{l}\text { Light brownish gray } \\
\text { nannofossil-radiolarian } \\
\text { ooze. }\end{array}$ & 10.8 & $\begin{array}{c}10.8 \\
(10.9)\end{array}$ & $\begin{array}{c}0.43 \\
(0.41)\end{array}$ & $\begin{array}{c}0.53 \\
(0.52)\end{array}$ & $\begin{array}{l}1.20 \\
(1.18)\end{array}$ & 607 & 19.51 & 2.51 & $\begin{array}{c}3.0 \\
(3.2)\end{array}$ & $\begin{array}{l}0.18 \\
(0.20)\end{array}$ & 606 & 35.2 & 32 & 7.5 \\
\hline $11-5$ & 98 & $\begin{array}{l}\text { Lower } \\
\text { Miocene }\end{array}$ & $\begin{array}{l}\text { White nannofossil chalk } \\
\text { ooze. }\end{array}$ & 10.8 & $\begin{array}{c}10.6 \\
(10.9)\end{array}$ & $\begin{array}{c}0.37 \\
(0.37)\end{array}$ & $\begin{array}{c}0.60 \\
(0.60)\end{array}$ & $\begin{array}{c}1.14 \\
(1.15)\end{array}$ & 601 & 19.52 & 2.34 & 3.6 & 0.22 & 603 & 35.0 & 31 & 7.4 \\
\hline \multicolumn{18}{|l|}{$70 \mathrm{~A}$} \\
\hline $1-2$ & 115 & $\begin{array}{l}\text { Lower } \\
\text { Miocene }\end{array}$ & $\begin{array}{l}\text { Yellowish-gray nannofossil- } \\
\text { radiolarian ooze. }\end{array}$ & 10.8 & 10.9 & 0.37 & 0.69 & 1.09 & 607 & 19.53 & 2.37 & 1.7 & 0.10 & 602 & 35.0 & 39 & 7.4 \\
\hline $5-6$ & 157 & $\begin{array}{l}\text { Upper } \\
\text { Oligocene }\end{array}$ & $\begin{array}{l}\text { Light greenish-gray } \\
\text { nannofossil-radiolarian } \\
\text { ooze. }\end{array}$ & 10.7 & 10.8 & 0.35 & 0.77 & 1.04 & 603 & 19.45 & 2.31 & 2.5 & 0.15 & 599 & 34.8 & 61 & 7.3 \\
\hline
\end{tabular}


TABLE 1 - Continued

\begin{tabular}{|c|c|c|c|c|c|c|c|c|c|c|c|c|c|c|c|c|}
\hline $\begin{array}{c}\text { Sample } \\
\text { Designation }\end{array}$ & $\begin{array}{l}\text { Depth } \\
\text { (m) }\end{array}$ & Age & Description & $\mathrm{Na}^{\mathrm{a}}$ & $\mathrm{Na}^{\mathrm{b}}$ & K & $\mathrm{Ca}$ & $\mathrm{Mg}$ & $\begin{array}{c}\text { Total } \\
\text { Cations } \\
\text { (meq/kg) }\end{array}$ & $\mathrm{Cl}$ & $\mathrm{SO}_{4}$ & $\underset{\text { (meq/kg) }}{\text { Alk. }}$ & $\mathrm{HCO}_{3}{ }^{\mathrm{c}}$ & $\begin{array}{c}\text { Total } \\
\text { Anions } \\
\text { (meq/kg) }\end{array}$ & Sum $^{d}$ & $\mathrm{H}_{2} \mathrm{O}(\%$ \\
\hline
\end{tabular}

Hole 70A - Continued

\begin{tabular}{|c|c|c|c|c|c|c|c|c|c|c|c|c|c|c|c|c|}
\hline $9-3$ & 189 & $\begin{array}{l}\text { Middle } \\
\text { Oligocene }\end{array}$ & $\begin{array}{l}\text { Light greenish-gray to pale } \\
\text { blue nannofossil-radiolarian } \\
\text { chalk. }\end{array}$ & 10.7 & $\begin{array}{c}10.9 \\
(10.7)\end{array}$ & $\begin{array}{c}0.30 \\
(0.32)\end{array}$ & $\begin{array}{c}0.96 \\
(0.98)\end{array}$ & $\begin{array}{c}0.98 \\
(0.96)\end{array}$ & 606 & 19.58 & 2.33 & $\begin{array}{l}1.5 \\
(2.1)\end{array}$ & $\begin{array}{c}0.09 \\
(0.13)\end{array}$ & 602 & 35.0 & 34 \\
\hline $13-2$ & 221 & $\begin{array}{l}\text { Middle } \\
\text { Oligocene }\end{array}$ & $\begin{array}{l}\text { Bluish white nannofossil } \\
\text { chalk. }\end{array}$ & 10.7 & $\begin{array}{c}10.7 \\
(10.4)\end{array}$ & $\begin{array}{c}0.26 \\
(0.27)\end{array}$ & $\begin{array}{c}1.17 \\
(1.16)\end{array}$ & $\begin{array}{c}0.86 \\
(0.84)\end{array}$ & 593 & 19.57 & 2.21 & $\begin{array}{c}2.2 \\
(2.1)\end{array}$ & $\begin{array}{c}0.13 \\
(0.13)\end{array}$ & 600 & 34.9 & 32 \\
\hline $28-1$ & 329 & $\begin{array}{l}\text { Upper } \\
\text { Eocene }\end{array}$ & $\begin{array}{l}\text { Very pale brown semi- } \\
\text { indurated radiolarian ooze. }\end{array}$ & 10.5 & 10.2 & 0.19 & 1.55 & 0.71 & 584 & 19.43 & 2.30 & 1.9 & 0.12 & 598 & 34.5 & 55 \\
\hline
\end{tabular}

\section{Site $71\left(4^{\circ} 28.3^{\prime} \mathrm{N}, 140^{\circ} 18.9^{\prime} \mathrm{W}\right.$, water depth $\left.4419 \mathrm{~m}\right)$}

Hole 71

\begin{tabular}{|c|c|c|c|c|c|c|c|c|c|c|c|c|c|c|c|c|}
\hline $4-3$ & 30 & $\begin{array}{l}\text { Upper } \\
\text { Miocene }\end{array}$ & $\begin{array}{l}\text { White and light brownish- } \\
\text { gray nannofossil-radiolarian } \\
\text { ooze. }\end{array}$ & 10.9 & $\begin{array}{c}10.9 \\
(10.8)\end{array}$ & $\begin{array}{c}0.41 \\
(0.43)\end{array}$ & $\begin{array}{c}0.43 \\
(0.42)\end{array}$ & $\begin{array}{l}1.25 \\
(1.24)\end{array}$ & 606 & 19.59 & 2.62 & $\begin{array}{l}2.3 \\
(2.4)\end{array}$ & $\begin{array}{c}0.14 \\
(0.15)\end{array}$ & 609 & 35.3 & 52 \\
\hline $12-3$ & 101 & $\begin{array}{l}\text { Middle } \\
\text { Miocene }\end{array}$ & $\begin{array}{l}\text { Very light gray nannofossil- } \\
\text { radiolarian ooze. }\end{array}$ & 10.8 & 10.9 & 0.42 & 0.60 & 1.09 & 605 & 19.63 & 2.23 & 2.6 & 0.16 & 603 & 35.0 & 34 \\
\hline $23-4$ & 203 & $\begin{array}{l}\text { Lower } \\
\text { Miocene }\end{array}$ & $\begin{array}{l}\text { Light gray to grayish-green } \\
\text { nannofossil-radiolarian } \\
\text { ooze. }\end{array}$ & 10.9 & 10.6 & 0.35 & 0.86 & 0.93 & 591 & 19.63 & 2.29 & 3.3 & 0.20 & 605 & 35.0 & 36 \\
\hline $30-3$ & 265 & $\begin{array}{l}\text { Lower } \\
\text { Miocene }\end{array}$ & $\begin{array}{l}\text { Bluish-white foraminiferal- } \\
\text { nannofossil ooze. }\end{array}$ & 10.9 & 11.0 & 0.35 & 0.96 & 0.85 & 606 & 19.65 & 1.93 & 2.4 & 0.15 & 603 & 35.1 & 32 \\
\hline $36-5$ & 323 & $\begin{array}{l}\text { Lower } \\
\text { Miocene }\end{array}$ & $\begin{array}{l}\text { Bluish-white foraminiferal- } \\
\text { nannofossil-radiolarian } \\
\text { chalk. }\end{array}$ & 10.9 & $\begin{array}{c}10.9 \\
(10.8)\end{array}$ & $\begin{array}{l}0.35 \\
(0.33)\end{array}$ & $\begin{array}{c}1.03 \\
(1.00)\end{array}$ & $\begin{array}{c}0.77 \\
(0.78)\end{array}$ & 596 & 19.66 & 2.08 & $\begin{array}{c}2.1 \\
(1.5)\end{array}$ & $\begin{array}{c}0.13 \\
(0.09)\end{array}$ & 600 & 34.9 & 28 \\
\hline $42-3$ & 375 & $\begin{array}{l}\text { Upper } \\
\text { Oligocene }\end{array}$ & $\begin{array}{l}\text { Bluish-white } \\
\text { nannofossil chalk. }\end{array}$ & 10.9 & 11.4 & 0.32 & 1.09 & 0.75 & 622 & 19.70 & 2.10 & 2.3 & 0.14 & 602 & 35.0 & 28 \\
\hline $48-3$ & 430 & $\begin{array}{l}\text { Upper } \\
\text { Oligocene }\end{array}$ & $\begin{array}{l}\text { Bluish-white to light gray } \\
\text { nannofossil chalk. }\end{array}$ & 10.9 & 11.0 & 0.34 & 1.14 & 0.71 & 603 & 19.70 & 2.06 & 1.9 & 0.12 & 600 & 35.0 & 28 \\
\hline
\end{tabular}

\section{Site $72\left(0^{\circ} 26.5^{\prime} \mathrm{N}, 138^{\circ} 52.0^{\prime} \mathrm{W}\right.$, water depth $\left.4326 \mathrm{~m}\right)$}

Hole 72

\begin{tabular}{|c|c|c|c|c|c|c|c|c|c|c|c|c|c|c|c|c|}
\hline $2-4$ & 64 & $\begin{array}{l}\text { Upper } \\
\text { Miocene }\end{array}$ & $\begin{array}{l}\text { Greenish and purple white } \\
\text { nannofossil ooze. }\end{array}$ & 10.9 & 10.7 & 0.39 & 0.44 & 1.22 & 598 & 19.46 & 2.53 & 3.4 & 0.21 & 605 & 35.1 & - \\
\hline $5-6$ & 219 & $\begin{array}{l}\text { Lower } \\
\text { Miocene }\end{array}$ & $\begin{array}{l}\text { Greenish-white } \\
\text { nannofossil ooze. }\end{array}$ & 10.8 & 10.8 & 0.40 & 0.50 & 1.15 & 600 & 19.42 & 2.35 & 4.0 & 0.24 & 601 & 34.9 & 32 \\
\hline $9-4$ & 335 & $\begin{array}{l}\text { Lower } \\
\text { Oligocene }\end{array}$ & White nannofossil ooze. & 10.8 & 10.9 & 0.37 & 0.50 & 1.16 & 604 & 19.52 & 2.30 & 3.4 & 0.21 & 602 & 34.9 & 34 \\
\hline
\end{tabular}


TABLE 1 - Continued

\begin{tabular}{|c|c|c|c|c|c|c|c|c|c|c|c|c|c|c|}
\hline $\begin{array}{c}\text { Sample } \\
\text { Designation }\end{array}$ & $\begin{array}{l}\text { Depth } \\
\text { (m) }\end{array}$ & Age & Description & $\mathrm{Na}^{\mathrm{a}}$ & $\mathrm{Na}^{\mathrm{b}}$ & K & $\mathrm{Ca}$ & $\mathrm{Mg}$ & $\begin{array}{c}\text { Total } \\
\text { Cations } \\
\text { (meq/kg) }\end{array}$ & $\mathrm{Cl}$ & $\mathrm{SO}_{4}$ & $\begin{array}{c}\text { Alk. } \\
\text { (meq/kg) }\end{array}$ & $\mathrm{HCO}_{3}{ }^{\mathrm{c}}$ & $\mathrm{Sum}^{\mathrm{d}} \mathrm{H}_{2} \mathrm{O}(\% \circ) p \mathrm{H}^{\mathrm{e}}$ \\
\hline
\end{tabular}

Hole 72 - Continued

72A-2-3 $21 \quad$ Pliocene

Pliocene Brownish and graying white

radiolarian ooze and

nannofossil-radiolarian

ooze.

\section{Site $73\left(1^{\circ} 54.6^{\prime} \mathrm{S}, 137^{\circ} 28.1^{\prime} \mathrm{W}\right.$, water depth $\left.4387 \mathrm{~m}\right)$}

Hole 73

\begin{tabular}{|c|c|c|c|c|c|c|c|c|c|c|c|c|c|c|c|c|}
\hline $2-3$ & 6 & $\begin{array}{l}\text { Pleisto- } \\
\text { cene }\end{array}$ & $\begin{array}{l}\text { Varicolored alternating } \\
\text { beds foraminiferal- } \\
\text { nannofossil-radiolarian } \\
\text { ooze. }\end{array}$ & 11.0 & 10.6 & 0.42 & 0.41 & 1.25 & 595 & 19.73 & 2.65 & 3.3 & 0.20 & 615 & 35.7 & 41 \\
\hline $6-4$ & 45 & $\begin{array}{l}\text { Lower } \\
\text { Pliocene }\end{array}$ & $\begin{array}{l}\text { Light bluish-gray and } \\
\text { grayish-green nannofossil- } \\
\text { radiolarian ooze. }\end{array}$ & 10.9 & 10.6 & 0.44 & 0.42 & 1.22 & 594 & 19.54 & 2.60 & 2.5 & 0.16 & 607 & 35.3 & 60 \\
\hline $12-3$ & 143 & $\begin{array}{l}\text { Lower } \\
\text { Miocene }\end{array}$ & $\begin{array}{l}\text { White to light gray } \\
\text { nannofossil chalk ooze. }\end{array}$ & 10.9 & 10.8 & 0.41 & 0.42 & 1.20 & 600 & 19.44 & 2.43 & 3.6 & 0.22 & 602 & 35.0 & 30 \\
\hline $20-5$ & 298 & $\begin{array}{l}\text { Upper } \\
\text { Eocene }\end{array}$ & $\begin{array}{l}\text { Yellowish brown semi- } \\
\text { indurated nannofossil- } \\
\text { radiolarian ooze. }\end{array}$ & 11.0 & 11.0 & 0.44 & 0.43 & 1.20 & 610 & 19.60 & 2.53 & 3.2 & 0.19 & 609 & 35.3 & - \\
\hline
\end{tabular}

Hole 74

\begin{tabular}{|c|c|c|c|}
\hline $2-2$ & 11 & $\begin{array}{l}\text { Upper } \\
\text { Miocene }\end{array}$ & $\begin{array}{l}\text { Dusky yellow brown } \\
\text { radiolarian ooze. }\end{array}$ \\
\hline 4-6 & 35 & $\begin{array}{l}\text { Lower } \\
\text { Miocene }\end{array}$ & $\begin{array}{l}\text { Very pale orange nanno- } \\
\text { fossil ooze. }\end{array}$ \\
\hline $6-5$ & 52 & $\begin{array}{l}\text { Upper } \\
\text { Oligocene }\end{array}$ & $\begin{array}{l}\text { Grayish-orange to } \\
\text { yellowish-brown } \\
\text { nannofossil ooze. }\end{array}$ \\
\hline $8-2$ & 67 & $\begin{array}{l}\text { Upper } \\
\text { Oligocene }\end{array}$ & $\begin{array}{l}\text { Grayish-orange } \\
\text { nannofossil ooze. }\end{array}$ \\
\hline
\end{tabular}

$\begin{array}{lllll}11.0 & 10.7 & 0.41 & 0.41 & 1.23\end{array}$

$\begin{array}{lllll}0.9 & 11.2 & 0.44 & 0.41 & 1.23\end{array}$

597

$19.63 \quad 2.59$

2.7

$19.53 \quad 2.64$

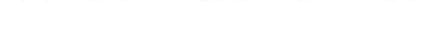

$\begin{array}{llllll}10.9 & 10.8 & 0.41 & 0.37 & 1.27 & 603\end{array}$

$\begin{array}{lll}19.53 & 2.64 \quad 2.9\end{array}$

$\begin{array}{lll}19.55 & 2.48 \quad 3.2\end{array}$

Site $75\left(12^{\circ} 31.0^{\prime} \mathrm{S}, 134^{\circ} 16.0^{\prime} \mathrm{W}\right.$, water depth $\left.4181 \mathrm{~m}\right)$

Hole 75

$\begin{array}{lllll}10.8 & 10.6 & 0.40 & 0.43 & 1.25\end{array}$

596

$19.42 \quad 2.64$ Miocene

Yellow nannoofossil ooze. 
TABLE 1 - Continued

\begin{tabular}{|c|c|c|c|c|c|c|c|c|c|c|c|c|c|c|c|c|c|}
\hline $\begin{array}{c}\text { Sample } \\
\text { Designation }\end{array}$ & $\begin{array}{l}\text { Depth } \\
\text { (m) }\end{array}$ & Age & Description & $\mathrm{Na}^{\mathrm{a}}$ & $\mathrm{Na}^{\mathrm{b}}$ & $\mathbf{K}$ & $\mathrm{Ca}$ & $\mathrm{Mg}$ & $\begin{array}{c}\text { Total } \\
\text { Cations } \\
\text { (meq } / \mathrm{kg})\end{array}$ & $\mathrm{Cl}$ & $\mathrm{SO}_{4}$ & $\begin{array}{c}\text { Alk. } \\
(\mathrm{meq} / \mathrm{kg})\end{array}$ & $\mathrm{HCO}_{3}{ }^{\mathrm{c}}$ & $\begin{array}{c}\text { Total } \\
\text { Anions } \\
(\mathrm{meq} / \mathrm{kg})\end{array}$ & Sum $^{d}$ & $\mathrm{H}_{2} \mathrm{O}(\%)$ & $p \mathrm{H}^{\mathrm{e}}$ \\
\hline \multicolumn{18}{|c|}{ Hole 75 - Continued } \\
\hline $4-5$ & 33 & $\begin{array}{l}\text { Upper } \\
\text { Oligocene }\end{array}$ & $\begin{array}{l}\text { Yellow nannofossil ooze, } \\
\text { trace amounts of } \\
\text { phillipsite. }\end{array}$ & 10.9 & 11.0 & 0.42 & 0.44 & 1.30 & 618 & 19.74 & 2.54 & 3.1 & 0.19 & 613 & 35.5 & 32 & 7.6 \\
\hline $9-6$ & 80 & $\begin{array}{l}\text { Lower } \\
\text { Oligocene }\end{array}$ & $\begin{array}{l}\text { Very dark grayish-brown } \\
\text { clay with nannofossils. }\end{array}$ & 11.0 & 10.6 & 0.44 & 0.45 & 1.20 & 593 & 19.63 & 2.62 & 2.2 & 0.13 & 610 & 35.5 & 39 & 7.7 \\
\hline
\end{tabular}

${ }^{\mathrm{a}}$ Sodium determined by differences between anions and cations excluding $\mathrm{Na}$.

bodium determined directly. Values in parentheses refer to separate samples carried through the entire sampling and analy tical processes. The total cation values shown are determined using these values and means of duplicate determinations, where available.

$\mathrm{C}_{\mathrm{HCO}}$ is calculated from total alkalinity, assuming this is entirely due to bicarbonate ion.

The sum incorporates the calculated Na values and means of replicate value where available. Minor constituents are not included but-with the exception of strontium in some samplesthey contribute less than $0.1 \%$ o to the sum.

$\mathrm{e}_{p \mathrm{H}}$ and water content are taken from Shipboard Summaries.

$\mathrm{f}_{\text {Duplicate values in parentheses. }}$ 
TABLE 2

Leg 8 Minor Constituents. Values in ppm. ${ }^{a}$

\begin{tabular}{|c|c|c|c|c|c|c|c|}
\hline $\begin{array}{l}\text { Sample } \\
\text { Designation }\end{array}$ & $\begin{array}{l}\text { Depth } \\
\text { (m) }\end{array}$ & Age & Description & $\mathrm{Sr}$ & $\mathrm{Ba}$ & $\operatorname{Si}(\text { col. })^{b}$ & $\mathrm{Li}$ \\
\hline \multicolumn{8}{|c|}{ Site $69\left(6^{\circ} 00.0^{\prime} \mathrm{N}, 152^{\circ} 51.9^{\prime} \mathrm{W}\right.$, water depth $4978 \mathrm{~m}$, South of Hawaii) } \\
\hline $69-3-3$ & 27 & $\begin{array}{l}\text { Middle } \\
\text { Miocene }\end{array}$ & $\begin{array}{l}\text { Dark yellowish-brown radio- } \\
\text { larian ooze w/beds of } \\
\text { nannofossil-radiolarian ooze. }\end{array}$ & 8.6 & $<0.1$ & 26 & 0.22 \\
\hline $5-3$ & 120 & $\begin{array}{l}\text { Lower } \\
\text { Oligocene }\end{array}$ & $\begin{array}{l}\text { Pale yellow brown and pale } \\
\text { brown nannofossil-radiolarian } \\
\text { oozes. }\end{array}$ & 9.5 & 0.10 & 27 & 0.18 \\
\hline $69 A-3-2$ & 81 & $\begin{array}{l}\text { Middle } \\
\text { Oligocene }\end{array}$ & $\begin{array}{l}\text { White to yellow brown } \\
\text { nannofossil-radiolarian ooze. }\end{array}$ & $8.6(9.3)$ & $<0.1(<0.1)$ & $25(24)$ & $0.19(0.20)$ \\
\hline $8-6$ & 143 & $\begin{array}{l}\text { Lower } \\
\text { Oligocene }\end{array}$ & $\begin{array}{l}\text { White w/beds of pale brown } \\
\text { nannofossil-radiolarian ooze. }\end{array}$ & $8.8(8.6)$ & $0.10(0.10)$ & $28(36)$ & $0.18(0.16)$ \\
\hline $11-2$ & 216 & $\begin{array}{l}\text { Middle } \\
\text { Eocene }\end{array}$ & Dark brown radiolarian ooze. & $10.2(8.8)$ & $0.10(<0.1)$ & $29(32)$ & $0.18(0.16)$ \\
\hline \multicolumn{8}{|c|}{$\begin{array}{l}\text { Site } 70\left(6^{\circ} 20.1^{\prime} \mathrm{N}, 140^{\circ} 21.7^{\prime} \mathrm{W} \text {, water depth } 5059 \mathrm{~m} \text {, }\right. \\
\text { Continuation of North to South Profile Started on Leg } 5)\end{array}$} \\
\hline $70-3-1$ & 17 & $\begin{array}{l}\text { Middle } \\
\text { Miocene }\end{array}$ & $\begin{array}{l}\text { Dark grayish-brown radiolarian } \\
\text { ooze. }\end{array}$ & 7.9 & $<0.1(<0.1)$ & $11(12)$ & $0.16(0.18)$ \\
\hline $7-2$ & 55 & $\begin{array}{l}\text { Lower } \\
\text { Miocene }\end{array}$ & $\begin{array}{l}\text { Light brownish-gray } \\
\text { nannofossil-radiolarian ooze. }\end{array}$ & 13.7 & $<0.1(<0.1)$ & $21(26)$ & $0.25(0.21)$ \\
\hline $11-5$ & 98 & $\begin{array}{l}\text { Lower } \\
\text { Miocene }\end{array}$ & $\begin{array}{l}\text { White nannofossil chalk } \\
\text { ooze. }\end{array}$ & 9.1 & $0.1(<0.1)$ & $24(25)$ & $0.12(0.13)$ \\
\hline $70 \mathrm{~A}-1-2$ & 115 & $\begin{array}{l}\text { Lower } \\
\text { Miocene }\end{array}$ & $\begin{array}{l}\text { Yellowish-gray nannofossil } \\
\text { radiolarian ooze. }\end{array}$ & 14.6 & $<0.1$ & 19 & 0.19 \\
\hline $5-6$ & 157 & $\begin{array}{l}\text { Upper } \\
\text { Oligocene }\end{array}$ & $\begin{array}{l}\text { Light greenish-gray } \\
\text { nannofossil-radiolarian ooze. }\end{array}$ & 13.8 & $<0.1$ & 18 & 0.16 \\
\hline $9-3$ & 189 & $\begin{array}{l}\text { Middle } \\
\text { Oligocene }\end{array}$ & $\begin{array}{l}\text { Light greenish-gray to pale blue } \\
\text { nannofossil-radiolarian ooze. }\end{array}$ & $16.7(18.0)$ & $0.10(<0.1)$ & $26(26)$ & $0.16(0.17)$ \\
\hline $13-2$ & 221 & $\begin{array}{l}\text { Middle } \\
\text { Oligocene }\end{array}$ & Bluish-white nannofossil chalk. & $18.2(20.2)$ & $<0.1(<0.1)$ & $30(16)$ & $0.17(0.17)$ \\
\hline $28-1$ & 329 & $\begin{array}{l}\text { Upper } \\
\text { Eocene }\end{array}$ & $\begin{array}{l}\text { Very pale brown semi- } \\
\text { indurated radiolarian ooze. }\end{array}$ & 18.6 & $<0.1$ & 38 & 0.22 \\
\hline \multicolumn{8}{|c|}{ Site $71\left(4^{\circ} 28.3^{\prime} \mathrm{N}, 140^{\circ} 18.9^{\prime} \mathrm{W}\right.$, water depth $\left.4419 \mathrm{~m}\right)$} \\
\hline $71-4-3$ & 30 & $\begin{array}{l}\text { Upper } \\
\text { Miocene }\end{array}$ & $\begin{array}{l}\text { White and light brownish-gray } \\
\text { nannofossil-radiolarian ooze. }\end{array}$ & $22.0(25.0)$ & $<0.1(0.12)$ & $11(12)$ & $0.23(0.24)$ \\
\hline $71-12-3$ & 101 & $\begin{array}{l}\text { Middle } \\
\text { Miocene }\end{array}$ & $\begin{array}{l}\text { Very light gray nannofossil } \\
\text { radiolarian ooze. }\end{array}$ & 51.0 & 0.17 & 24 & 0.13 \\
\hline 234 & 203 & $\begin{array}{l}\text { Lower } \\
\text { Miocene }\end{array}$ & $\begin{array}{l}\text { Light gray to grayish-green } \\
\text { nannofossil-radiolarian ooze. }\end{array}$ & 95.0 & $<0.1$ & 30 & 0.11 \\
\hline $30-3$ & 265 & $\begin{array}{l}\text { Lower } \\
\text { Miocene }\end{array}$ & $\begin{array}{l}\text { Bluish-white foraminiferal } \\
\text { nannofossil ooze. }\end{array}$ & 0.8 & 0.1 & - & 0.06 \\
\hline $36-5$ & 323 & $\begin{array}{l}\text { Lower } \\
\text { Miocene }\end{array}$ & $\begin{array}{l}\text { Bluish-white foraminiferal } \\
\text { nannofossil-radiolarian chalk. }\end{array}$ & $96.0(84.0)$ & $0.1(<0.1)$ & $42(26)$ & 0.10 \\
\hline
\end{tabular}


TABLE 2 - Continued

\begin{tabular}{|c|c|c|c|c|c|c|c|}
\hline $\begin{array}{c}\text { Sample } \\
\text { Designation }\end{array}$ & $\begin{array}{l}\text { Depth } \\
\text { (m) }\end{array}$ & Age & Description & $\mathrm{Sr}$ & $\mathrm{Ba}$ & $\mathrm{Si}(\mathrm{col} .)^{\mathrm{b}}$ & $\mathrm{L}$ \\
\hline \multicolumn{8}{|c|}{ Site 71 - Continued } \\
\hline $42-3$ & 375 & $\begin{array}{l}\text { Upper } \\
\text { Oligocene }\end{array}$ & $\begin{array}{l}\text { Bluish-white nannofossil } \\
\text { chalk. }\end{array}$ & 119.0 & 0.11 & 24 & 0.07 \\
\hline $48-3$ & 430 & $\begin{array}{l}\text { Upper } \\
\text { Oligocene }\end{array}$ & $\begin{array}{l}\text { Bluish-white to light } \\
\text { gray nannofossil chalk. }\end{array}$ & 92.0 & 0.13 & 24 & 0.08 \\
\hline \multicolumn{8}{|c|}{ Site $72\left(0^{\circ} 26.5^{\prime} \mathrm{N}, 138^{\circ} 52.0^{\prime} \mathrm{W}\right.$, water depth $\left.4326 \mathrm{~m}\right)$} \\
\hline $72-2-4$ & 64 & $\begin{array}{l}\text { Upper } \\
\text { Miocene }\end{array}$ & $\begin{array}{l}\text { Greenish and purple } \\
\text { white nannofossil ooze. }\end{array}$ & 37.0 & $<0.1$ & 16 & 0.14 \\
\hline $5-6$ & 219 & $\begin{array}{l}\text { Lower } \\
\text { Miocene }\end{array}$ & $\begin{array}{l}\text { Greenish-white nannofossil } \\
\text { ooze. }\end{array}$ & 50.0 & 0.10 & 21 & 0.09 \\
\hline 94 & 335 & $\begin{array}{l}\text { Lower } \\
\text { Oligocene }\end{array}$ & White nannofossil ooze. & 41.0 & 0.10 & 20 & 0.11 \\
\hline $72 A-2-3$ & 21 & Pliocene & $\begin{array}{l}\text { Brownish and grayish-white } \\
\text { radiolarian ooze and nannofossil- } \\
\text { radiolarian ooze. }\end{array}$ & 19.4 & $<0.1$ & 18 & 0.19 \\
\hline \multicolumn{8}{|c|}{ Site $73\left(1^{\circ} 54.6^{\prime} \mathrm{S}, 137^{\circ} 28.1^{\prime} \mathrm{W}\right.$, water depth $\left.4387 \mathrm{~m}\right)$} \\
\hline $73-2-3$ & 6 & $\begin{array}{l}\text { Pleisto- } \\
\text { cene }\end{array}$ & $\begin{array}{l}\text { Varicolored alternating beds } \\
\text { foraminiferal-nannofossil- } \\
\text { radiolarian ooze. }\end{array}$ & 12.9 & $<0.1$ & - & 0.22 \\
\hline $6-4$ & 45 & $\begin{array}{l}\text { Lower } \\
\text { Pliocene }\end{array}$ & $\begin{array}{l}\text { Light bluish-gray and grayish } \\
\text { green nannofossil-radiolarian } \\
\text { ooze. }\end{array}$ & 16.5 & 0.11 & 25 & 0.21 \\
\hline $12-3$ & 143 & $\begin{array}{l}\text { Lower } \\
\text { Miocene }\end{array}$ & $\begin{array}{l}\text { White to light gray } \\
\text { nannofossil chalk ooze. }\end{array}$ & 15.8 & $<0.1$ & 14 & 0.20 \\
\hline $20-5$ & 298 & $\begin{array}{l}\text { Upper } \\
\text { Eocene }\end{array}$ & $\begin{array}{l}\text { Yellowish-brown semi- } \\
\text { indurated nannofossil- } \\
\text { radiolarian ooze. }\end{array}$ & 14.4 & 0.10 & 22 & 0.24 \\
\hline \multicolumn{8}{|c|}{ Site $74\left(6^{\circ} 14.2^{\prime} \mathrm{S}, 136^{\circ} 05.8^{\prime} \mathrm{W}\right.$, water depth $\left.4431 \mathrm{~m}\right)$} \\
\hline $74-2-2$ & 11 & $\begin{array}{l}\text { Upper } \\
\text { Miocene }\end{array}$ & $\begin{array}{l}\text { Dusky yellow brown radio- } \\
\text { ooze. }\end{array}$ & 8.8 & $<0.1$ & 25 & 0.29 \\
\hline $4-6$ & 35 & $\begin{array}{l}\text { Lower } \\
\text { Miocene }\end{array}$ & $\begin{array}{l}\text { Very pale orange nannofossil } \\
\text { ooze. }\end{array}$ & 9.0 & 0.10 & 12 & 0.21 \\
\hline $6-5$ & 52 & $\begin{array}{l}\text { Upper } \\
\text { Oligocene }\end{array}$ & $\begin{array}{l}\text { Grayish-orange to yellowish- } \\
\text { brown nannofossil ooze. }\end{array}$ & 8.5 & $<0.1$ & 14 & 0.25 \\
\hline $8-2$ & 67 & $\begin{array}{l}\text { Upper } \\
\text { Oligocene }\end{array}$ & $\begin{array}{l}\text { Grayish-orange nannofossil } \\
\text { ooze. }\end{array}$ & 9.8 & 0.1 & 17 & 0.29 \\
\hline
\end{tabular}


TABLE 2 - Continued

\begin{tabular}{|c|c|c|c|c|c|c|c|}
\hline $\begin{array}{c}\text { Sample } \\
\text { Designation }\end{array}$ & $\begin{array}{l}\text { Depth } \\
\text { (m) }\end{array}$ & Age & Description & $\mathrm{Sr}$ & $\mathrm{Ba}$ & $\operatorname{Si}(\text { col. })^{b}$ & $\mathrm{Li}$ \\
\hline \multicolumn{8}{|c|}{ Site $75\left(12^{\circ} 31.0^{\prime} \mathrm{S}, 134^{\circ} 16.0^{\prime} \mathrm{W}\right.$, water depth $\left.4181 \mathrm{~m}\right)$} \\
\hline $75-2-2$ & 10 & $\begin{array}{l}\text { Lower } \\
\text { Miocene }\end{array}$ & Yellow nannofossil ooze. & 8.3 & 0.14 & 5 & 0.28 \\
\hline $4-5$ & 33 & $\begin{array}{l}\text { Upper } \\
\text { Oligocene }\end{array}$ & $\begin{array}{l}\text { Yellow nannofossil ooze, } \\
\text { trace amounts of phillipsite. }\end{array}$ & 7.9 & 0.14 & 10 & 0.31 \\
\hline $9-6$ & 80 & $\begin{array}{l}\text { Lower } \\
\text { Oligocene }\end{array}$ & $\begin{array}{l}\text { Very dark grayish-brown } \\
\text { clay with nannofossils. }\end{array}$ & 9.3 & 0.20 & 9 & 0.45 \\
\hline
\end{tabular}

${ }^{\mathrm{a}}$ Duplicate values for $\mathrm{Sr}, \mathrm{Ba}$, and $\mathrm{Li}$ in parentheses.

${ }^{\mathrm{b}}$ Values in parentheses represent colorimetric analyses.

and simultaneous decreases in magnesium concentrations occur with depth (Figure 1). Extraordinary increases in strontium concentration occur in Sites 71 and 72 .

(3) Moderate decreases in sulfate concentrations occur in Sites 70, 71 and 72.

(4) Silica concentrations typically range between 20 and $30 \mathrm{ppm}$, a tenfold or greater increase over values obtained in the open ocean.

(5) The $p \mathrm{H}$ values are somewhat lower (7.3) in the anomalous holes than in the others.

(6) Water-content decreases characteristically occur in the first few meters or tens of meters of cores in pelagic sediments. However, below a few tens of meters no significant trends could be observed. It should be pointed out that occasional high water content, as shown here, may be due to disturbance of the cores. Packaged core subsamples carefully chosen to represent the least disturbed sediments have not yet been analyzed here. Below 50 meters (sediment penetration) water content ranging between 30 and 35 per cent seems to be typical for the biogenic oozes, with no significant decrease with depth observable.

As observed on previous open ocean legs, the conservative constituents-chlorine ( $\mathrm{Cl}$ ) and sodium (Na)showed few deviations from values characteristic of present-day bottom waters beyond those which may be attributed to manipulation of the sediments and pore fluids on board ship, and analytical scatter. If any significant change occurs, it may be a small $(<1$ per cent) increase in chlorinity with depth in Hole 71. Similar marginal tendencies have been noted in previous legs.

\section{DISCUSSION}

Major interest centers on the origin of the large departures in calcium, magnesium and strontium concentrations with depth, from those characteristic of sea water values. In the absence of evaporites, clayey sediments in geosynclinal accumulations from the Gulf of Mexico (Manheim and Bischoff, 1969), western Atlantic continental margin (Chan and Manheim, 1970), Vema Trench (Sayles et al., 1970), and continental margin off the coast of Oregon (Manheim et al., 1970) have been shown to take up magnesium and potassium, leading to deficits in the pore fluids. Similar tendencies, though less pronounced, have been observed in pore fluids obtained from piston-cored sediments (Bruevich, 1965, and references cited therein). In the current leg, Sites 70 and 71 are overwhelmingly dominated by siliceous and calcareous nannofossil remains-Radiolaria and coccolithophorids, in particular. Yet, they show decreases in magnesium and potassium similar to anomalies heretofore associated with terrigenous clays. Further, calcium is dramatically enriched in Sites 70 and 71 , whereas strontium is very high in Sites 71 and 72 (to 113 and $50 \mathrm{ppm}$, respectively) but only moderately enriched in Site 70 .

The following factors were examined for their possible genetic relationship to the anomalies:

(1) Water depth. All sites in Leg 8 are at depths greater than 4000 meters, and no consistent relationship between pore fluid chemistry among these holes or previous Pacific holes can be determined.

(2) General lithology. Leg 8 sites are dominated by biogenic oozes; and, siliceous and calcareous nannoplankton material was characteristic of all sites from which pore fluids were obtained. Although quantitative 


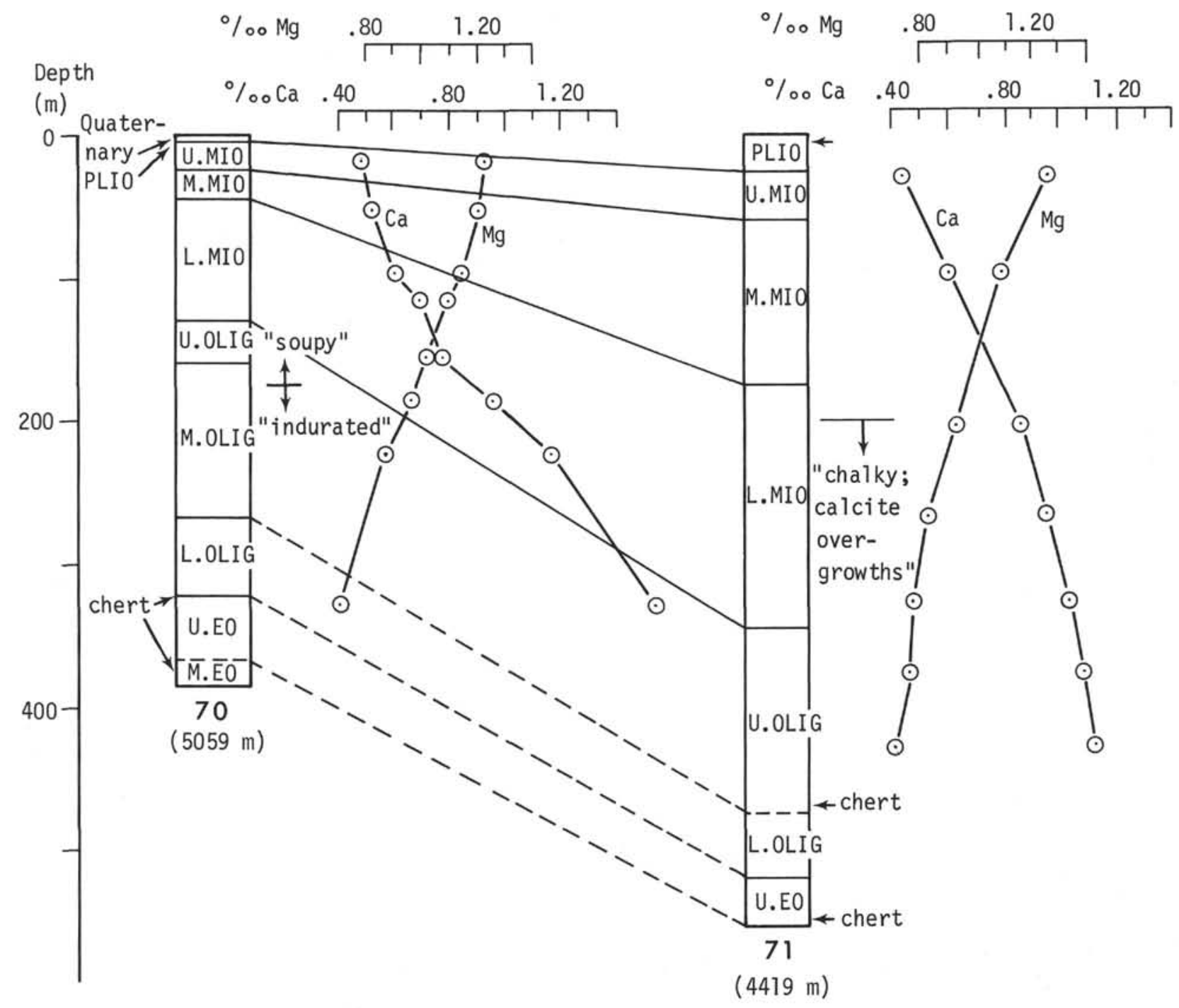

Figure 1. Distribution of calcium and magnesium in pore fluid of Sites 70 and 71 . Stratigraphy is taken from preliminary site descriptions (Shipboard Logs). Both sites represent relatively rapid deposition rates.

data on the proportion of clay in the anomalous holes is not yet available, it seems to be very small. Such differences occurring in gross lithology seem incapable of accounting for observed effects.

(3) Age of sediments. Ages are coherent in Sites 69 through 75 , and offer no distinctive pattern in the anomalous strata.

(4) Temperature or physical gradients. Although heat flow measurements were attempted, the efforts were not successful. However, there is no reason to presume that thermal gradients vary markedly over the area in question. The fact that excess potassium was observed in the upper portions of Sites 70 and 71, and declined with depth would at first appear to agree with the observations of Mangelsdorf et al. (1969) and Bischoff et al. (1970), regarding the loss of potassium $(\mathrm{K})$ and gain of magnesium $(\mathrm{Mg})$ by sediments as they are warmed prior to squeezing on board. However, in this case the sediments apparently contain few exchangeable materials capable of releasing potassium. One material which has not yet been sufficiently checked for its exchange behavior is amorphous silica of pelagic sediments. Zaitseva (1965) claims that pelagic biogenic debris does not have appreciable exchange capacity. Her analyses indicated that some nonclayey sediments in the Pacific were capable of total exchange capacity close to that of clayey samples, but she attributed this behavior to the presence of such minerals as allophane and zeolites, particularly phillipsite. 
(5) Rate of accumulation of sediments. As may be seen in Figures 2 and 3, those sites which exhibit the most marked anomalies, Sites 70,71 and 72 , also demonstrate the most rapid accumulation rates. Though not shown in the figures, one can also note qualitatively that local variations in the anomaly trends are often associated with intra-site changes in accumulation rate for the sediments. To view the relationships more quantitatively, Figure 3 shows plots of mean losses and gains for magnesium and strontium in pore fluids against mean accumulation rate for each site taken as a unit. Calcium (not shown) has a slope and scatter more like that of magnesium than the very strong relationship shown by strontium. Potassium, on the other hand, has little consistent relationship with accumulation rate in these cores. In part, the anomalies are reminiscent of the high strontium pore fluids found in biogenic oozes from sites in the East Carolina Basin and Ontong Java Plateau (Leg 7, Sayles and Manheim, 1971).
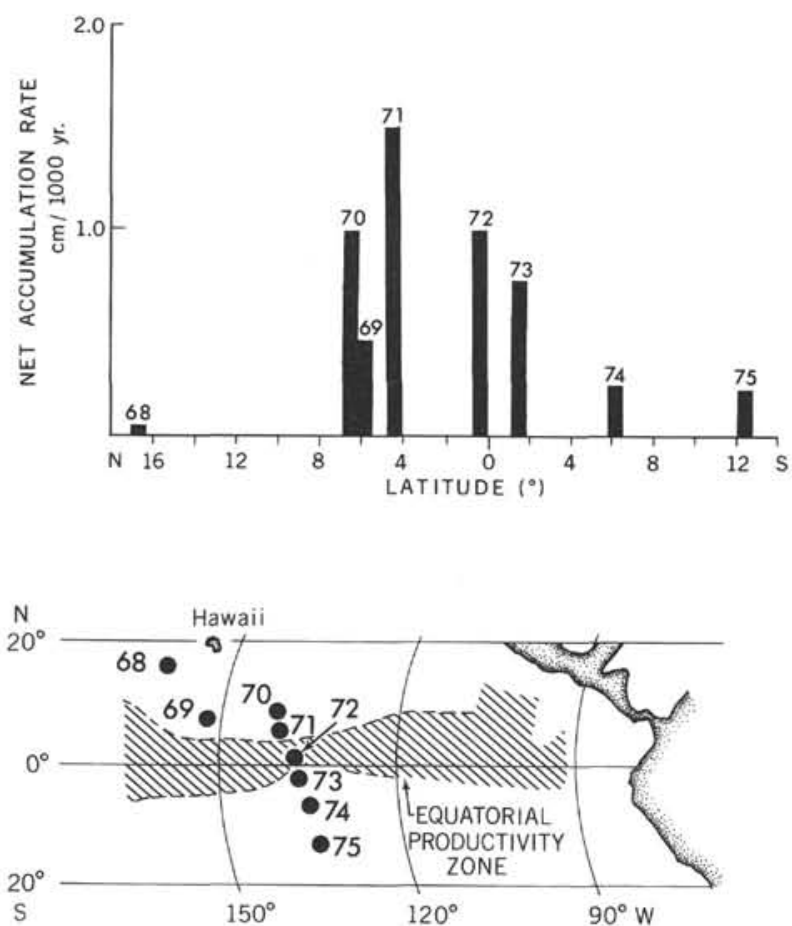

Figure 2. Location and accumulation rates for sites in Leg 8. Productivity boundaries are taken from Heezen and Hollister, 1971.

(6) Diagenesis and recrystallization of skeletal remains. One phenomenon clearly related to the pore fluid anomalies is the reported evidences of recrystallization and induration phenomena in the sediments of Sites 70 and 71 . Carbonate overgrowths on discoasters and other organisms were singled out for comment in the lithologic Hole Summaries. Radiolaria were cited as being well-preserved in Site 72 down to Oligocene layers, where corrosion was noted. Thus, there is evidence that both leaching of solid phases and formation of new authigenic phases is going on in the sediments. Table 3 shows relative gains and losses in calcium, magnesium and strontium, expressed in milliequivalents per kilogram fluid. The question remains: what phases are being leached and what is taking up magnesium (and potassium)?

TABLE 3

Changes in Composition of Pore Fluids in Sites 70 and 71, Expressed as Differences from Mean Sea Water (mg-eq. $/ \mathrm{kg})^{\mathrm{a}}$

\begin{tabular}{cccccc}
\hline Site & $\begin{array}{c}\text { Depth } \\
(\mathrm{m})\end{array}$ & $\mathrm{K}$ & $\mathrm{Ca}$ & $\mathrm{Mg}$ & $\mathrm{Sr}$ \\
\hline 70 & 17 & +1.3 & +2.3 & -4.1 & \\
& 55 & +1.0 & +5.8 & -7.3 & $+(0.07)$ \\
& 98 & -0.4 & +9.3 & -10.5 & +0.01 \\
& 155 & -0.4 & +13.7 & -15.5 & +0.08 \\
& 157 & -1.0 & +17.7 & -19.5 & +0.07 \\
& 189 & -2.0 & +27.6 & -25.3 & +0.10 \\
& 221 & -3.2 & +37.1 & -35.1 & +0.13 \\
& 329 & -5.0 & +56.4 & -46.7 & +0.12 \\
71 & 30 & +0.8 & +0.8 & -1.5 & +0.18 \\
& 101 & +0.8 & +9.2 & -15.6 & +0.49 \\
& 203 & -1.0 & +21.9 & -28.6 & +1.00 \\
& 265 & -1.0 & +27.1 & -35.1 & +0.89 \\
& 323 & -1.2 & +29.5 & -40.9 & +0.94 \\
& 375 & -1.6 & +33.5 & -43.5 & +1.27 \\
& 430 & -1.2 & +36.0 & -46.7 & +0.96 \\
\hline
\end{tabular}

According to available data, pure Recent-sub-Recent coccolith ooze and fresh foraminiferal carbonate have about 0.15 per cent strontium and 0.14 per cent magnesium (Table 4). These values should be compared against those from fossil equivalents which have not been exposed to meteoric waters on land or unusual conditions, in order to evaluate net losses and gains in skeletal remains with time. Published chemical data on these constituents in solids of the Deep Sea Drilling Program cores are not yet available, but evidence from JOIDES drillings (Manheim, 1971, cited in Table 4) off Florida indicates that older Tertiary coccolith oozes have more magnesium and less strontium than the sub-Recent materials. Further, particularly marked decreases in strontium were observed in sediments showing evidence of consolidation and lithification (less than 0.08 per cent strontium, compared with about 0.12 per cent strontium for unconsolidated oozes). 


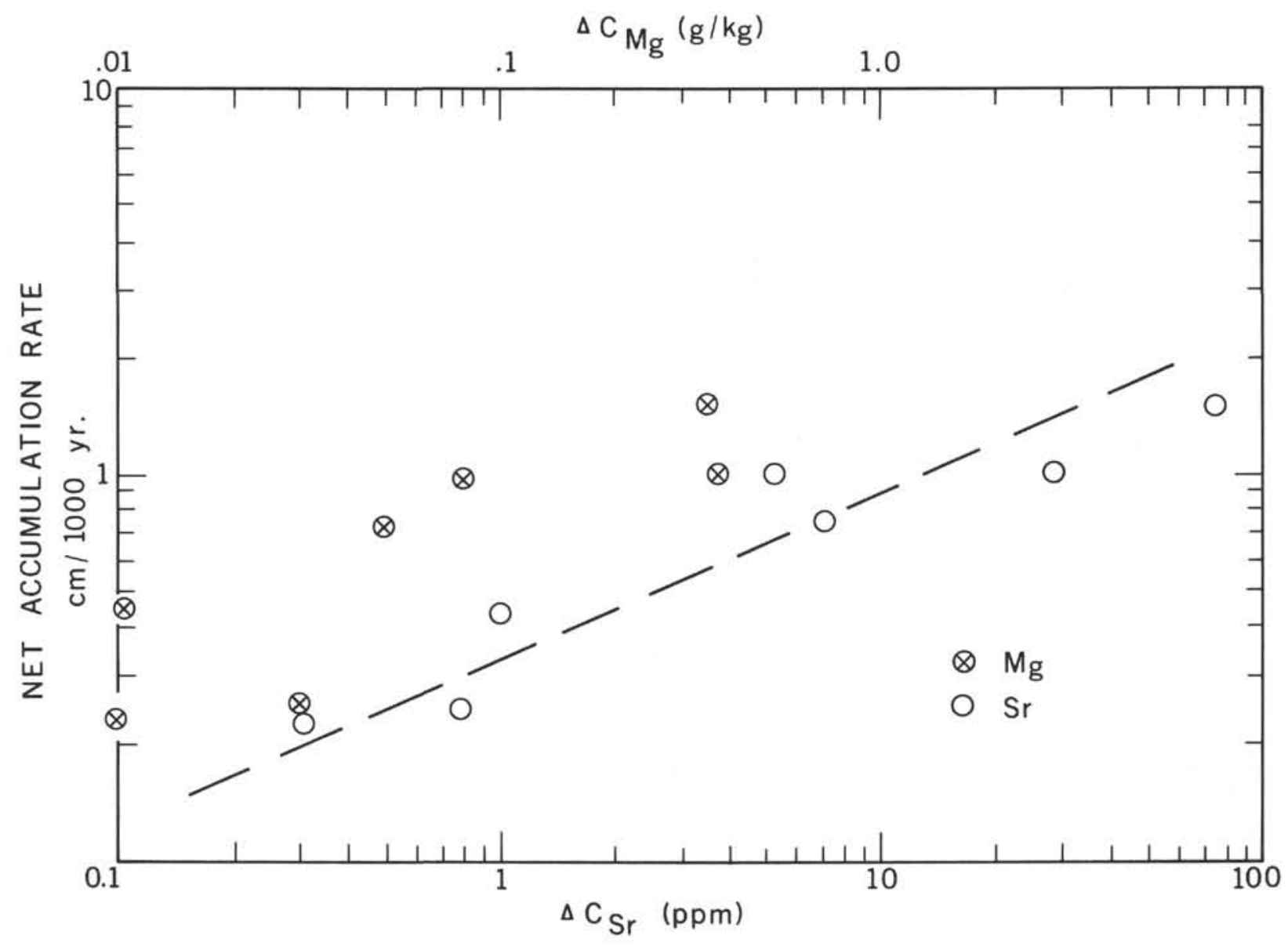

Figure 3. Plot of $\mathrm{Mg}$ and $\mathrm{Sr}$ anomalies against accumulation rate (mean) for all analyzed sites. $\Delta C$ is obtained by subtracting the value for mean ocean water from the mean concentration of the given species in the pore fluids of each site.

Strontium enrichments in the pore water appear anomalous. The excess $\mathrm{Ca}: \mathrm{Sr}$ ratios (ions in pore water in excess of normal oceanic values) in Site 71 are less than 10:1, compared with $\mathrm{Ca}: \mathrm{Sr}$ ratios in coccolith or foraminiferal ooze of more than 250:1. The highest strontium content known in organisms reported to be preserved in sediments is about 1.0 per cent $\mathrm{SrO}$ in nonmolluscan aragonite (Milliman, 1971). Acantharian Radiolaria are known to contain celestite $\left(\mathrm{SrSO}_{4}\right)$ (for example, Hollande and Cachon-Enjumet, 1963), but since this phase is greatly undersaturated in sea-water, these organisms dissolve rapidly on death, and are not reported as having been preserved in sediments (Kling, 1970 ). Virtually nothing is known about the composition of non-acantharian Radiolaria, except that they have siliceous skeletons and, therefore, are unlikely to contain large strontium concentrations. Finally, preliminary analyses of organisms from sediments in Site 71 by scanning electron microprobe (Kevex) revealed no particles having unusual strontium concentrations.
In the absence of organisms or special phases containing very large strontium concentrations we must assume that carbonate ooze is being dissolved and a calcite phase containing less strontium and more magnesium is being reprecipitated. Emiliani (1955) concluded that there was virtually no magnesium in shell carbonate; amounts being detected in core samples were due to contamination with terrigenous detritus. However, the data of Krinsley (1960) and Lipps and Ribbe (1967) contradict this view. Without taking the loss of excess metals due to diffusion and totally reprecipitated-forming oozes having concentrations like those given for the Tertiary oozes in Table 4-to account for the strontium anomalies. Larger proportions of the sediments would have to be dissolved and reprecipitated to account for magnesium. These processes are still unclear, since thermodynamics would predict that calcite should not recrystallize to a higher magnesium calcite in a closed system (for example, Weyl, 1965). Kinetics may play a role, and 
TABLE 4

Strontium and Magnesium Carbonate Organisms Prominent in Oozes, and Selected Sediments

\begin{tabular}{|c|c|c|c|}
\hline Description & $\mathrm{Sr}$ & $\mathrm{Mg}$ & Source \\
\hline $\begin{array}{l}\text { Coccolith ooze, gravity core from tropical } \\
\text { Atlantic, } 2 \text { meter length. } \\
\text { Mean of three analyses }(7,97,169 \mathrm{~cm}) \text {. }\end{array}$ & 0.150 & 0.11 & Thompson and Bowrn, 1969 \\
\hline $\begin{array}{l}\text { Foraminiferal carbonate from Caribbean Sea } \\
\text { (piston core). } \\
\text { Mean of } 35 \text { analyses. }\end{array}$ & 0.160 & 0.15 & Wangersky and Joensuu, 1964 \\
\hline $\begin{array}{l}\text { Foraminiferal carbonate, Atlantic Ocean, } \\
2 \text { cores. } \\
\text { More than } 50 \text { analyses. }\end{array}$ & 0.160 & 0.13 & Wangersky and Joensuu, 1964 \\
\hline $\begin{array}{l}\text { Planktonic foraminifera, tops of Atlantic cores } \\
\text { (Globigerinoides rubra, Globigerina inflata, and } \\
\text { Globorotalia truncatulinoides). } \\
\text { Mean of analyses from Caribbean, Azores, } \\
\text { Bermuda, Cape Verde and Canary Is. }\end{array}$ & 0.150 & 0.16 & Krinsley, 1960 \\
\hline $\begin{array}{l}\text { Site 3, JOIDES drilling program, off South- } \\
\text { eastern U. S. Coccolith-foraminiferal ooze- } \\
\text { Upper Miocene to Middle Eocene age } \\
\text { (175 meters total depth). } \\
\text { Mean } 12 \text { samples (HCl-leachable fraction). }\end{array}$ & 0.094 & 0.28 & Manheim, 1971 \\
\hline
\end{tabular}

the introduction of carbon dioxide $\left(\mathrm{CO}_{2}\right)$ on a continuous basis may also be a factor in causing significant dissolution of carbonate and adjusting the degree of supersaturation in discrete layers of the strata. Organic ligands may also shift stability fields in favor of magnesian calcites (Kitano and Kanamori, 1966).

Once the major reactions are sorted out, corrections due to ancillary factors may bring observation into harmony with theory. Such factors include differences in diffusion constants, and the porosity (ratio of liquid to solid) in the sediments.

\section{REFERENCES}

Bischoff, J. L., Greer, R. E. and Luistro, A. O., 1970. Composition of interstitial waters of marine sediments: temperature of squeezing effect. Science. 167,1245 .

Bruevich, S. V., 1965. Khimiya gruntovykh rastvorov Tikhogo okeana (Chemistry of pore fluids in sediments of the Pacific Ocean). Pt. 2, In Khimiya Tikhogo okeana, Izdat. "Nauka”., Moscow. 261.

Chan, K-M, and Manheim, F. T., 1970. Interstitial water studies on small core samples, Deep Sea Drilling Project, Leg 2. In Initial Reports of the Deep Sea Drilling Project, Volume II. Washington (U. S. Government Printing Office), 367.
Emiliania, C., 1955. Mineralogy and chemical composition of the tests of certain pelagic foraminifera. Micropaleontology. 1, 377.

Heezen, B. C. and Hollister, C. D., 1971. The Face of the Deep. (Oxford University Press) (in press).

Hollande, A. and Cachon-Enjumet, M., 1963. Sur la constitution chimique des spicules d'Acanthaires. Bull. de l'Inst. Ocean. Monaco. 60, 1263.

Kitano, Y. and Kanamori, N., 1966. Synthesis of magnesian-calcite at low temperatures and pressures. Geochem. J. 1, 1.

Krinsley, D., 1960. Trace elements in the tests of planktonic foraminifera. Micropaleontology. 6, 297 .

Lipps, J. H. and Ribbe, P. H., 1967. Electron-probe microanalysis of planktonic foraminifera. J. Paleontolog. 41, 492.

Mangelsdorf, P. C., Jr., Wilson, T. R. S. and Daniell, E., 1969. Potassium enrichments in interstitial waters of recent marine sediments. Science. $65,171$.

Manheim, F. T. and Bischoff, J. L., 1969. Geochemistry of pore waters from Shell Oil Co., drill holes on the continental slope of the northern Gulf of Mexico. Chem. Geol. 4, 63.

Manheim, F. T., Chan, K-M. and Sayles, F. L., 1970. Interstitial water studies on small core samples, Deep Sea Drilling Project, Leg 5. In Initial Reports of the Deep Sea Drilling Project, Volume V. Washington (U.S. Government Printing Office), 501 . 
Milliman, J. D., 1971. Marine Carbonates. New York (Appleton Century Crofts, Inc.) (in press).

Sayles, F. L., Manheim, F. T. and Chan, K-M., 1970. Interstitial water studies on small core samples, Leg 4. Initial Reports of the Deep Sea Drilling Project, Volume IV. Washington (U. S. Government Printing Office), 401.

Sayles, F. L. and Manheim, F. T., 1971. Interstitial water studies on small core samples, Leg 7. In Initial Reports of the Deep Sea Drilling Project, Volume VIII. Washington (U.S. Government Printing Office) (in press).
Thompson, G. and Bowen, V. T., 1969. Analyses of coccolith ooze from the deep tropical Atlantic. $J$. Marine Res. 27, 32.

Wangersky, P. J. and Joensuu, O., 1964. Strontium, magnesium, and manganese in fossil foraminiferal carbonates. J. Geol. 72, 477.

Weyl, P. K., 1965. In Studies in Tropical Oceanography. F. M. Bayer et al. (Eds.) (5), 178.

Zaitseva, E. D., 1965. Emkost' obmena i obmennye kationy osadkov Tikhogo okeana (Cation exchange capacity of sediments from the Pacific Ocean). In Khimiya Tikhogo okeana, Izdat. "Nauka", Moscow. S. V. Bruevich (Ed.), 271. 